\title{
Expression and Clinical Value of miR- 185 and miR-424 in Patients with Acute Ischemic Stroke
}

\author{
Chunxuan Guol,* \\ Yanping $\mathrm{Yao}^{2, *}$ \\ Qi Li' \\ Yuanjie Gao' \\ Hongyan $\mathrm{Cao}^{3}$ \\ 'Department of Neurology, People's \\ Hospital of Danzhou, Danzhou, Hainan, \\ People's Republic of China; ${ }^{2}$ Department \\ of Pharmacy, Suzhou Xiangcheng People's \\ Hospital, Suzhou, Jiangsu, People's \\ Republic of China; ${ }^{3}$ Department of \\ Clinical Laboratory, Suzhou TCM \\ Hospital Affiliated to Nanjing University \\ of Chinese Medicine, Suzhou, Jiangsu, \\ People's Republic of China \\ *These authors contributed equally to \\ this work
}

Correspondence: Hongyan Cao Department of Clinical Laboratory, Suzhou TCM Hospital Affiliated to

Nanjing University of Chinese Medicine,

18 Yangsu Road, Suzhou, 215006, People's

Republic of China

$\mathrm{Tel} / \mathrm{Fax}+8618861332277$

Email 8I3233I2@qq.com
Background: To investigate the expression of serum miR-185 and miR-424 in patients with acute ischemic stroke (AIS) and their predictive value. A total of 142 patients with AIS and 50 healthy controls were enrolled.

Methods: According to the modified Rankin scale (mRS) score, AIS patients were divided into the good prognosis group and the poor prognosis group. Based on the National Institutes of Health Stroke Scale (NIHSS) score, AIS patients were divided into the mild group, the moderate group, and the severe group. RT-qPCR was used to determine the expression. ROC curve and Pearson correlation analysis were adopted to predict poor prognosis and analyze the correlation between the expression and NIHSS, mRS score.

Results: Compared with the control group, the expression of miR-185 and miR-424 in the AIS group was significantly higher $(P<0.01)$. Similarly, significantly higher expressions could be found in the poor prognosis group and the severe group $(P<0.01)$. The ROC curve revealed that the optimal cut-off values were 2.14 and 4.08, respectively. The area under the ROC curve $(0.928,95 \%$ CI: $0.870-0.993)$ was the largest, with sensitivity and specificity of $92.0 \%$ and $85.7 \%$. Pearson correlation analysis showed that their expression was positively correlated with NIHSS score and mRS score in AIS patients $(r=0.735,0.802$, 0.796, 0.873, $P<0.01)$.

Conclusion: There are two factors related to the up-regulated expression of serum miR-185 and miR-424, one is the severity degree of neurological impairment of patients with AIS and the other is their prognosis. These two combined indicators can contribute to predicting the prognosis of AIS patients.

Keywords: acute ischemic stroke, miR-185, miR-424, prognostic evaluation

\section{Introduction}

Acute ischemic stroke (AIS) is a dangerous cerebrovascular disease that brings nonreversible damage to our brain. ${ }^{1}$ It brings great mental stress and economic burden on patients and their families, especially the middle-aged and elder people. ${ }^{2-4}$ AIS is always recurrent and a very complex disease. ${ }^{5}$ Therefore, finding a helpful biomarker for AIS prognosis is of great importance. microRNA (miRNA), a new type of gene regulation molecule, participates in the occurrence and development of stroke by regulating gene expression, and is expected to serve as a potential biomarker of AIS. ${ }^{6}$ Recent studies have found that miR-185 and miR-424 are involved in initiating a series of complex pathophysiological processes of stroke. ${ }^{7-10}$ They play a vital role in the damage of atherosclerotic plaque, neuronal destruction and repair, and local inflammation in the lesion. ${ }^{11,12}$ However, further exploration is still needed, since the mechanisms and potential therapeutic effects of miR-185 and miR-424 in AIS are not 
clear. Therefore, in this study, the expression of serum miR185 and miR-424 in patients with AIS was detected, and the predictive value of miR-185 and miR-424 in patients with AIS was analyzed, to provide a reference for the early treatment of patients with AIS.

\section{Materials and Methods Study Population}

One hundred and forty-two patients with AIS admitted to People's Hospital of Danzhou from January 1, 2019 to July 31, 2021 were enrolled, including 97 males and 45 females, aged 35 to $87(53.60 \pm 13.25)$ years. The inclusion criteria: (a) diagnosis based on "Chinese Stroke Association guidelines for clinical management of cerebrovascular disorders"; ${ }^{13}$ and (b) the patients were hospitalized within 24 hours of onset, and the ischemic lesions were revealed by CT or MRI. The exclusion criteria of patients are as follows: (a) patients with hemorrhagic stroke, traumatic brain injury, hypertensive encephalopathy, encephalitis, and other types of cerebrovascular diseases; (b) patients with cardiopulmonary disease, multiple sclerosis, malignant tumors, and blood system diseases; and (c) patients with consciousness disorder or mental symptoms. Fifty cases of healthy controls were selected as the control group, including 32 males and 18 females, aged from 40 to 75 (57.20 \pm 9.28$)$ years. This study was approved by the hospital ethics committee of People's Hospital of Danzhou. All patients signed informed consent before the experiment was conducted. According to the modified Rankin scale (mRS) score, AIS patients were divided into the good prognosis group and the poor prognosis group. Based on the National Institutes of Health Stroke Scale (NIHSS) score, AIS patients were classified into the mild group, the moderate group, and the severe group. The NIHSS score was applied to evaluating the degree of neurological impairment of patients. Among them, 42 cases were patients with mild neurological impairment (the mild group, score $<5$ ), 64 cases were patients with moderate neurological impairment (the moderate group, score 5-20), and 36 cases were patients with severe neurological impairment (the severe group, score $>20$ ). The general information of all patients at the time of admission was recorded, including age, gender, body mass index, basic diseases, hyperlipidemia, history of smoking and drinking.

\section{Primer Design and Synthesis}

miR-185 Primer Upstream sequence: 5'-ACGTATG CTACTGTGAGCTG-3', Downstream sequence: 5'-GAC TGCGAGCTAGCATCG-3'; miR-424 Primer Upstream sequence: 5'-GAGTACTCTATGACTGTCAG-3', Downst ream sequence: 5'-ATCGTACTAGATACTACG-3'.

\section{Detection of miR-I85 and miR-424}

A sample of $5 \mathrm{~mL}$ of venous blood was taken from every patient on admission to the hospital, and placed in a centrifuge tube without anticoagulant. Then, centrifuge at $2500 \mathrm{r} / \mathrm{min}$ for $15 \mathrm{~min}$ at room temperature, pipet 400 $\mu \mathrm{L}$ of the upper serum and add $1 \mathrm{~mL}$ of extractant (Trizol) into it, and store that at $-70{ }^{\circ} \mathrm{C}$. Real-time fluorescent quantitative polymerase chain reaction (RT-PCR) was performed on the ABI 7500 fluorescent quantitative PCR instrument. The reaction system was $20.00 \mu \mathrm{L}$, including $1.00 \mu \mathrm{L}$ TaqMan MicroRNA Assay, $1.33 \mu \mathrm{L}$ cDNA, 10.00 $\mu \mathrm{L}$ TaqMan $2 \times$ Universal PCR Master Mix, $7.67 \mu \mathrm{L} \mathrm{ddH}_{2}$ $\mathrm{O}$. The mixture was centrifuged and put into a quantitative PCR instrument. The condition of PCR amplified reaction was $95{ }^{\circ} \mathrm{C}$ pre-degeneration for 10 minutes, $95{ }^{\circ} \mathrm{C}$ degeneration for 15 seconds, $60{ }^{\circ} \mathrm{C}$ annealing for 60 seconds, and $95{ }^{\circ} \mathrm{C}$ extension for 2 seconds which cycle for 45 times. Take $\mathrm{U} 6$ as the internal reference; the relative expression of serum miR-185 and miR-424 were solved by the method of $2^{-\Delta \Delta \mathrm{Ct}}, \Delta \mathrm{Ct}=\mathrm{Ct}_{\text {target gene }}-\mathrm{Ct}_{\mathrm{U} 6}$.

\section{Statistical Analysis}

In the analysis, SPSS 12.0 was applied, and the measurement data were represented by $\mathrm{X} \pm \mathrm{S}$, and the comparison of the means of two independent samples was conducted by $t$-test. The counting data were expressed as the percentage (\%) and were performed by $\chi^{2}$ test. ROC curve and Pearson correlation analysis was also applied. $P<0.05$ indicated the difference was statistically significant.

\section{Results}

\section{The Expression of Serum miR-185 and miR-424}

There was no significant difference in gender, body mass index (BMI), basic diseases, atrial fibrillation, smoking history, drinking history, heart rate, systolic blood pressure, and diastolic blood pressure between the good prognosis group and the poor prognosis group $(P>0.05)$ (Table 1). Compared with the control group, the expression of miR-185 and miR-424 in the AIS group was 
Table I Clinical Characteristics of the Study Population

\begin{tabular}{|c|c|c|c|c|}
\hline Variable & AIS Group $(n=142)$ & Cerebral Infarction $(n=44)$ & Control $(n=50)$ & $P$ \\
\hline Age (mean $\pm S D)$ & $52.40 \pm 2.35$ & $52.80 \pm 2.14$ & $52.15 \pm 2.83$ & 0.758 \\
\hline BMI $\left(\mathrm{kg} / \mathrm{m}^{2}\right)$ & $23.78 \pm 2.34$ & $23.18 \pm 2.14$ & $23.60 \pm 2.78$ & 0.316 \\
\hline \multicolumn{5}{|l|}{ Gender } \\
\hline Man & $82(57.7)$ & $24(54.5)$ & $30(60.0)$ & 0.824 \\
\hline Woman & $60(42.3)$ & $20(45.5)$ & $20(30.0)$ & \\
\hline \multicolumn{5}{|c|}{ History of Diabetes, n (\%) } \\
\hline Yes & $61(43.0)$ & $21(47.7)$ & $23(46.0)$ & 0.760 \\
\hline No & $81(57.0)$ & $23(52.3)$ & $27(54.0)$ & \\
\hline \multicolumn{5}{|c|}{ History of Hypertension, n (\%) } \\
\hline Yes & $85(59.9)$ & $27(6 \mid .4)$ & $31(62.0)$ & 0.624 \\
\hline No & $57(40.1)$ & $17(38.6)$ & $19(38.0)$ & \\
\hline \multicolumn{5}{|c|}{ Hyperlipidemia, n (\%) } \\
\hline Yes & $82(57.7)$ & $30(68.2)$ & $32(64.0)$ & 0.514 \\
\hline No & $60(42.3)$ & $14(3 \mid .8)$ & $18(36.0)$ & \\
\hline \multicolumn{5}{|c|}{ History of Drinking, n (\%) } \\
\hline Yes & $55(38.7)$ & $16(36.4)$ & $17(34.0)$ & 0.698 \\
\hline No & $87(6 \mid .3)$ & $28(63.6)$ & $33(66.0)$ & \\
\hline \multicolumn{5}{|c|}{ History of Smoking, n (\%) } \\
\hline Yes & 89 (62.7) & $35(79.5)$ & $38(76.0)$ & 0.114 \\
\hline No & $53(37.3)$ & $9(20.5)$ & $12(24.0)$ & \\
\hline
\end{tabular}

Abbreviation: AIS, acute ischemic stroke.

significantly higher $(P<0.01)$ (Figure 1$)$. Similarly, significantly higher expressions could be found in the poor prognosis group and the severe group $(P<0.01)$ (Figures 2 and 3). Furthermore, the expression levels of serum miR-185 and miR-424 in the large area cerebral infarction group were significantly higher than those in the medium area cerebral infarction group and small area cerebral infarction group $(P<0.01)$ (Figure 4).

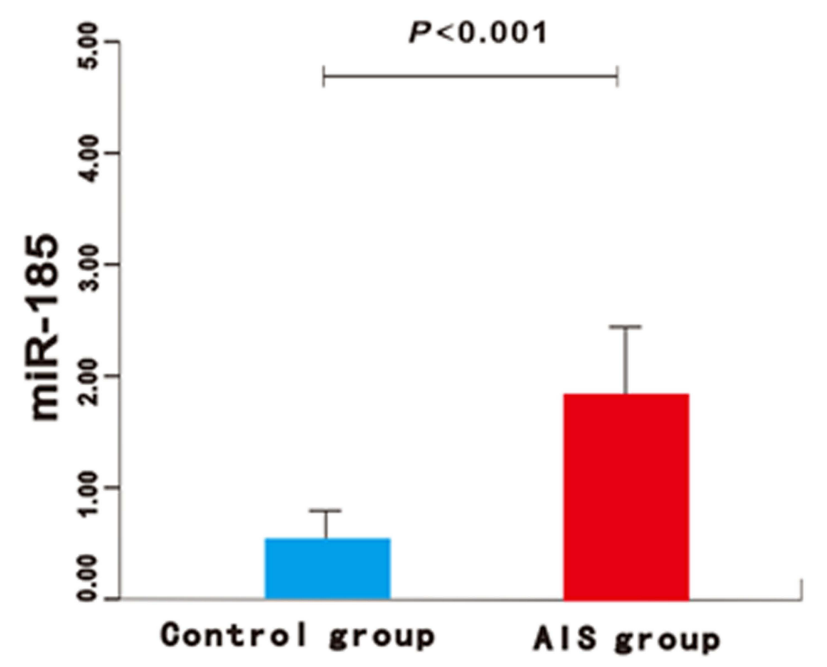

\section{ROC Curve}

The optimal cut-off values of the expression of serum miR-185 and miR-424 for predicting poor prognosis of AIS patients were 2.14 and 4.08, respectively. The AUC of the two combined predictors for poor prognosis of AIS patients was $0.928(0.870 \sim 0.993)$, which was significantly higher than that of single miR-185 [0.795 (0.737 0.856)] and single miR-424 [0.841 (0.785 0.897)], the differences

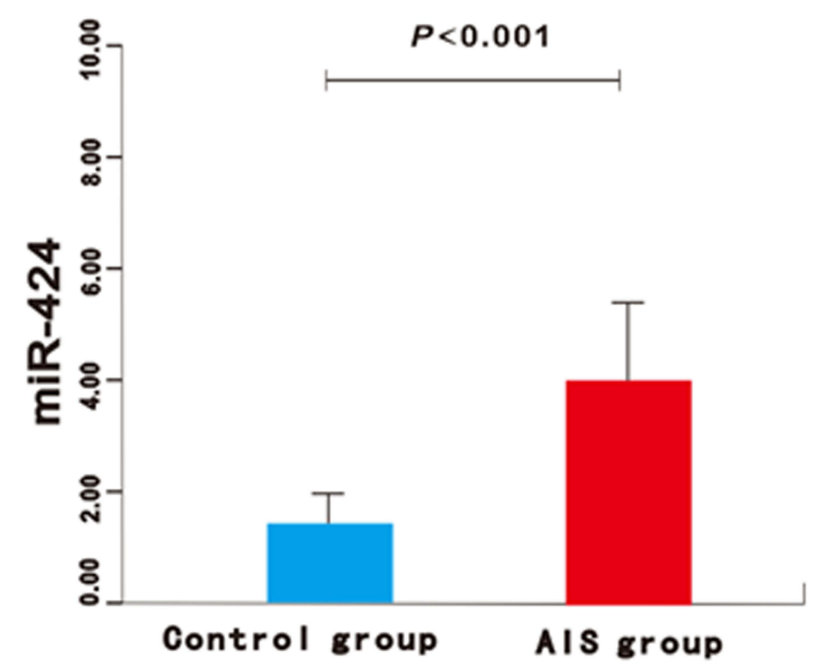

Figure I The expression of miR-185 and miR-424 in the AIS group and control group. Abbreviation: AIS, acute ischemic stroke. 

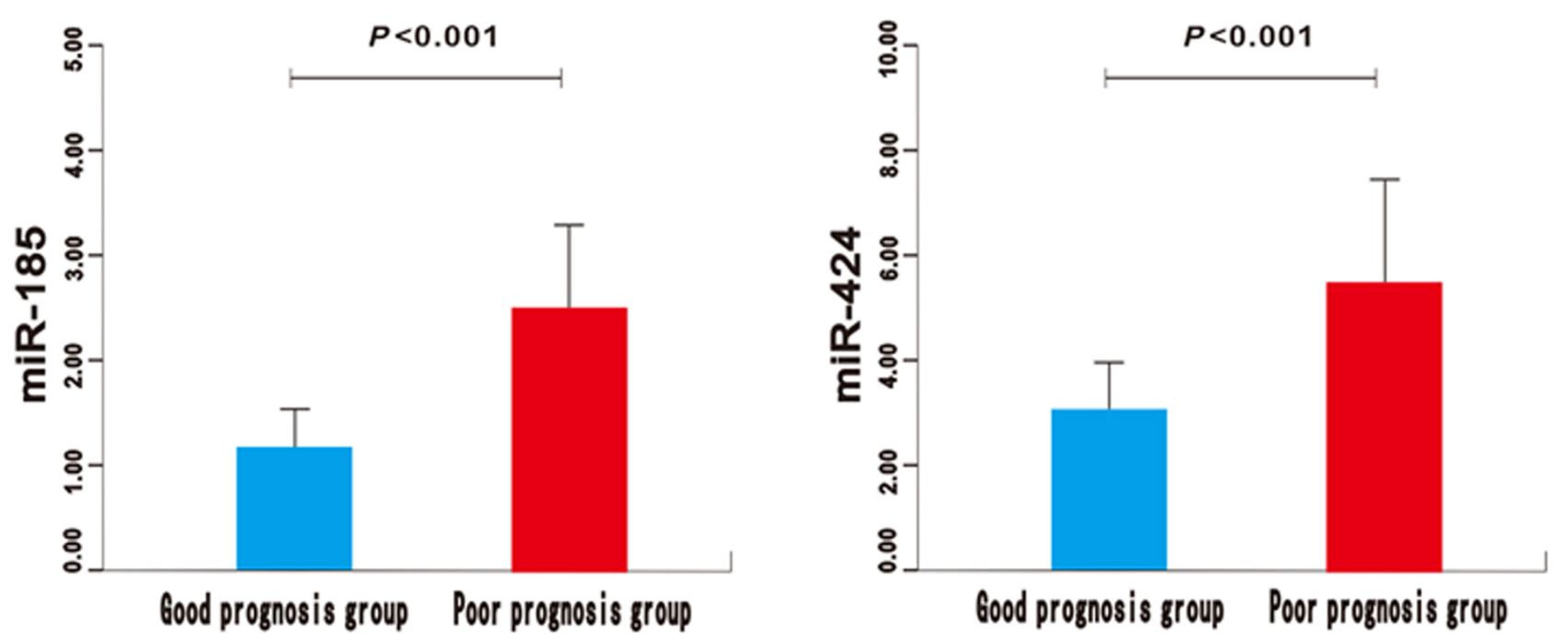

Figure 2 The expression of miR-185 and miR-424 in poor prognosis group and good prognosis group of AIS patients. Abbreviation: AIS, acute ischemic stroke.
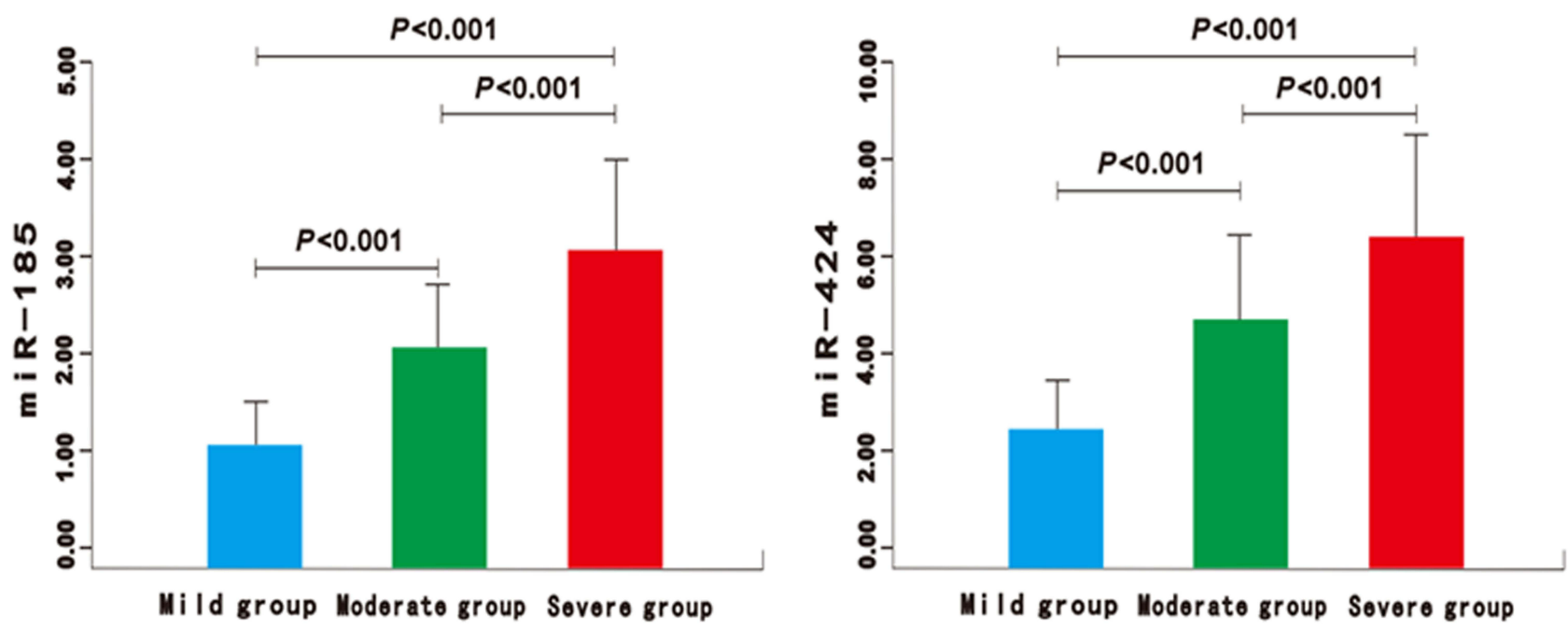

Figure 3 The expression of miR-185 and miR-424 in the mild, moderate, and severe group of AIS patients. Abbreviation: AIS, acute ischemic stroke.

were statistically significant $(Z=7.118,5.214, P<0.05)$, and the sensitivity and specificity were $92.7 \%$ and $86.5 \%$, respectively (Table 2 and Figure 5).

\section{Pearson Correlation Analysis}

Pearson correlation analysis showed that the expression of serum miR-185 was positively related to NIHSS score and $\mathrm{mRS}$ score $(r=0.735,0.802, \quad P<0.01)$ (Figure 6). Similarly, the expression of serum miR-424 was positively related to NIHSS score and mRS score ( $r=0.796,0.873, P<0.01)$ (Figure 7).

\section{Discussion}

AIS is a common vascular disease of the central nervous system, which can trigger a series of ischemic cascade pathological events, and finally lead to the apoptosis and necrosis of cerebrovascular endothelial cells and nerve cells. Inflammatory reaction, endothelial dysfunction, and atherosclerosis play a vital role in the pathophysiological process of AIS. ${ }^{14}$ miRNA, composed of 18-25 nucleotides in length, is an endogenous single-stranded non-coding RNA molecule. By affecting the expression of target genes, it participates in the initiation of a series of complex 


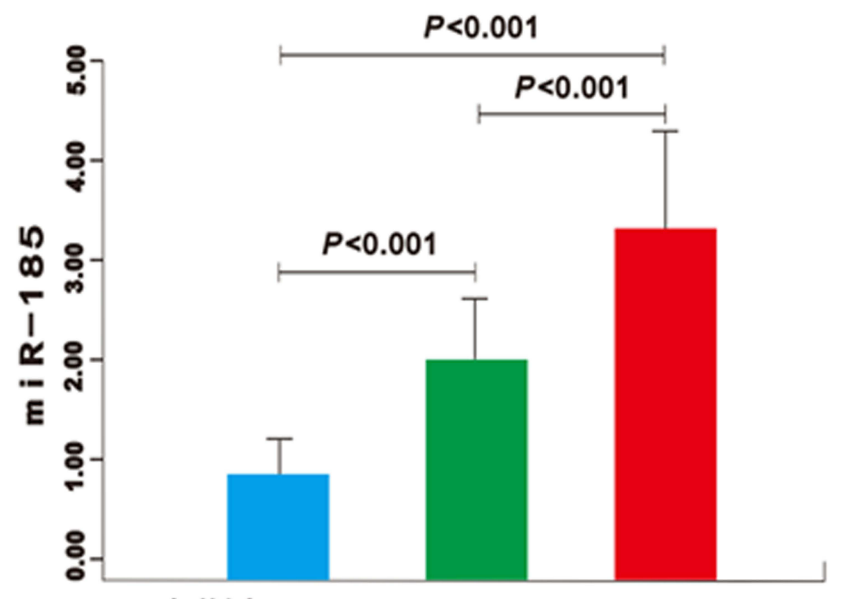

Sull infarct group lediun infarct group Large infarct group

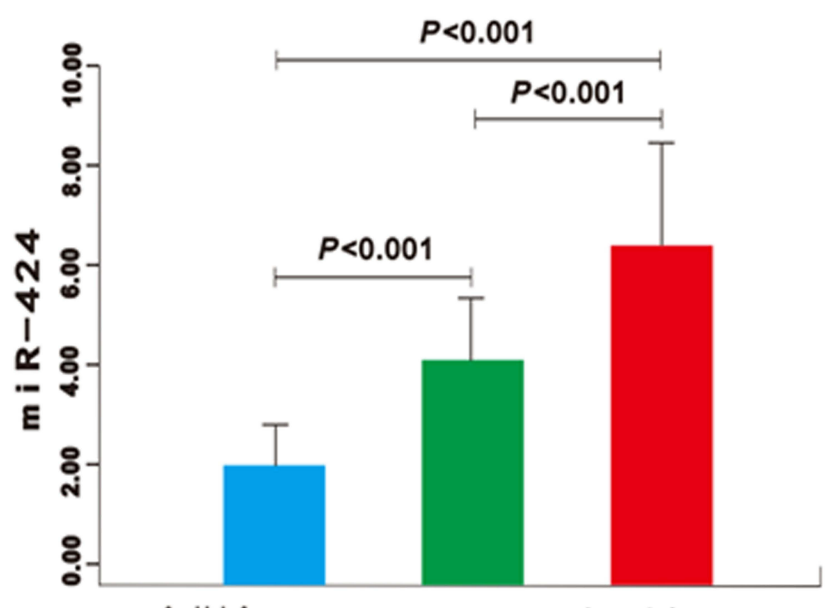

Sull infarct group lediun infarct group Large infarct group

Figure 4 The expression of miR-185 and miR-424 in small, medium, and large area cerebral infarction group of AIS patients. Abbreviation: AIS, acute ischemic stroke.

pathophysiological processes of AIS. ${ }^{15}$ Numerous studies have reported that miRNAs participate in degrading extracellular matrix, regulating cell apoptosis in plaques, regulating vascular endothelial function and vascular remodeling. miRNAs also can affect the formation and rupture of atherosclerotic plaques, and are involved in neuron destruction, repair, and local inflammatory reaction. miRNA may provide a new target for the treatment of AIS. ${ }^{16,17} \mathrm{Xu}$ et $\mathrm{al}^{18}$ found that the differential expression of miRNAs in AIS may be involved in the release of

Table 2 Clinical Characteristics of the Good Prognosis Group and Poor Prognosis Group

\begin{tabular}{|c|c|c|c|}
\hline Variable & Good Prognosis $(n=89)$ & Poor Prognosis $(n=53)$ & $\mathbf{P}$ \\
\hline Age $($ mean $\pm S D)$ & $51.48 \pm 9.35$ & $57.15 \pm 8.63$ & 0.006 \\
\hline BMI $\left(\mathrm{kg} / \mathrm{m}^{2}\right)$ & $23.28 \pm 2.24$ & $23.45 \pm 2.36$ & 0.584 \\
\hline Heart rate (beats/min) & $95.20 \pm 7.15$ & $96.36 \pm 7.40$ & 0.473 \\
\hline Systolic blood pressure $(\mathrm{mmHg})$ & $143.26 \pm 10.28$ & $145.40 \pm 10.37$ & 0.751 \\
\hline Diastolic blood pressure $(\mathrm{mmHg})$ & $85.73 \pm 7.26$ & $86.48 \pm 7.42$ & 0.557 \\
\hline NIHSS (score) & $10.50 \pm 3.25$ & $17.62 \pm 3.58$ & $<0.001$ \\
\hline mRS (score) & $1.42 \pm 0.28$ & $4.17 \pm 0.56$ & $<0.001$ \\
\hline \multicolumn{4}{|l|}{ Gender } \\
\hline Man & 58 & 39 & 0.297 \\
\hline Woman & 31 & 14 & \\
\hline \multicolumn{4}{|l|}{ History of Diabetes, n (\%) } \\
\hline Yes & $20(22.5)$ & $14(26.4)$ & 0.594 \\
\hline No & $69(77.5)$ & $39(73.6)$ & \\
\hline \multicolumn{4}{|l|}{ History of Hypertension, n (\%) } \\
\hline Yes & $27(30.3)$ & $19(35.8)$ & 0.561 \\
\hline No & $62(69.7)$ & $34(64.2)$ & \\
\hline \multicolumn{4}{|l|}{ History of Drinking, n (\%) } \\
\hline Yes & $37(41.6)$ & $25(47.2)$ & 0.515 \\
\hline No & $52(58.4)$ & $28(52.8)$ & \\
\hline \multicolumn{4}{|l|}{ History of Smoking, n (\%) } \\
\hline Yes & $29(32.3)$ & $15(28.3)$ & 0.594 \\
\hline No & $60(67.7)$ & $38(71.7)$ & \\
\hline \multirow[t]{2}{*}{ Atrial fibrillation, $\mathrm{n}(\%)$} & $7(7.9)$ & $5(9.4)$ & 0.745 \\
\hline & $82(92.1)$ & $58(90.6)$ & \\
\hline
\end{tabular}

Abbreviations: NIHSS, National Institutes of Health Stroke Scale; mRS, modified Rankin scale. 


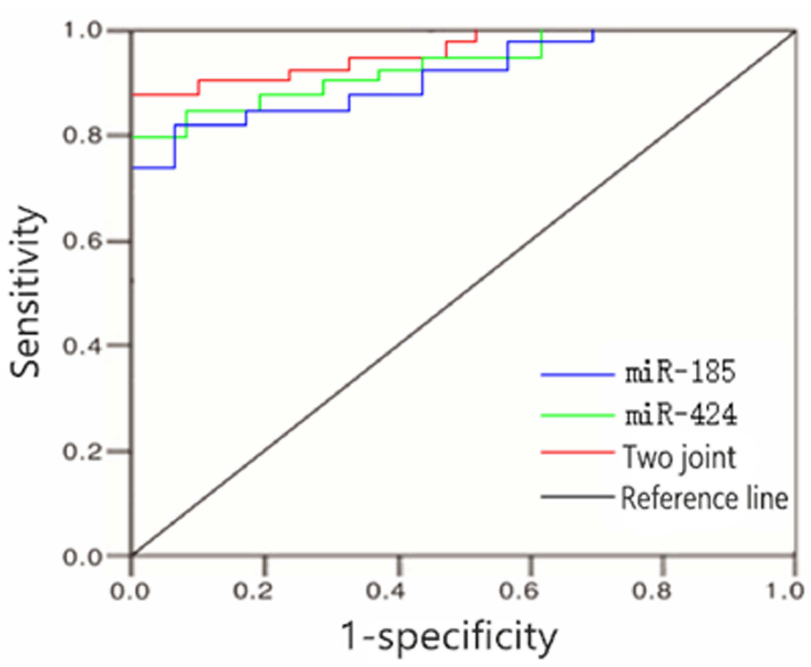

Figure 5 ROC curve for predicting poor prognosis of AIS patients by the expression of serum miR-185 and miR-424.

Abbreviation: AIS, acute ischemic stroke.

inflammatory mediators and endothelial cell dysfunction. This may also promote the formation and rupture of atherosclerotic plaque and affect the occurrence of stroke. Other studies pointed out that miRNA can identify the $3^{\prime}$ noncoding region of the target gene by complementary base pairing, degrade the target messenger RNA, or inhibit the translation of the target messenger RNA. It can participate in the regulation of the pathophysiological process of AIS as well, such as atherosclerosis, cerebral edema, and cerebral ischemia-reperfusion damage. ${ }^{19}$

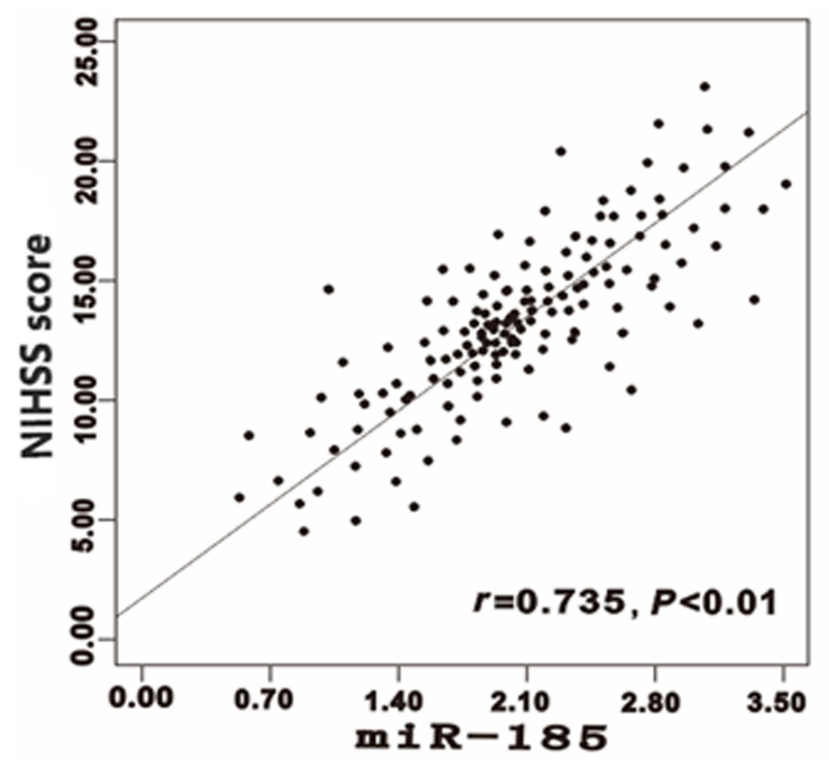

The present study suggests that the up-regulated expression of serum miR-185 and miR-424 may be involved in the pathogenesis of AIS. Moreover, there are differences in the expression of serum miR-185 and miR424 in patients with different degrees of neurological impairment. The more severe the neurological impairment, the more obvious the increased expression of serum miR185 and miR-424. This conclusion suggests that the increased expression of serum miR-185 and miR-424 is strongly associated with poor prognosis, and can indicate the severity of neurological impairment and may become biomarkers of AIS patients. The research by Gacon et $\mathrm{al}^{20}$ showed that miRNA was closely linked with the pathogenesis of AIS. Wu et $\mathrm{al}^{21}$ found that the expression of miRNA was closely linked with the degree of neurological impairment in stroke patients. Thus, the expression of miRNA can be used as a biological indicator for evaluating the degree of neurological impairment of AIS. The ROC curve indicated that the optimal cut-off values of the expression of serum miR-185 and miR-424 for predicting poor prognosis of AIS patients were 2.14 and 4.08, respectively. The area under the curve of the two combined indicators for predicting was the largest, with relatively higher sensitivity and specificity. Jin and Xing's ${ }^{22}$ research concluded that the expression of serum miR-185 in patients with AIS was significantly higher and was positively linked with NIHSS score. miR-185 can be used as an independent biomarker of AIS risk and has a good

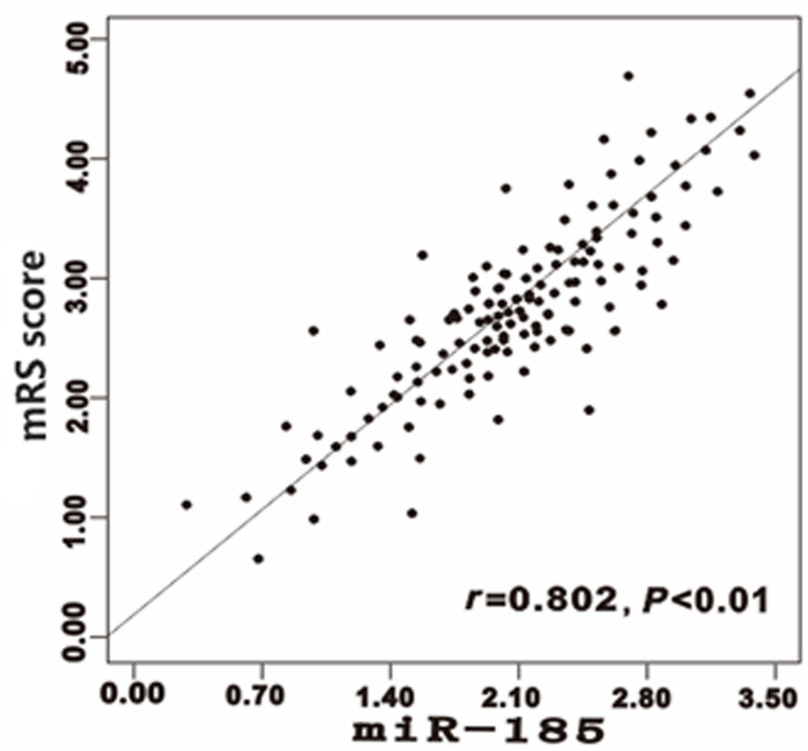

Figure 6 Correlations between the expression of serum miR-185 and NIHSS, mRS score in patients with AIS.

Abbreviations: AIS, acute ischemic stroke; NIHSS, National Institutes of Health Stroke Scale; mRS, modified Rankin scale. 

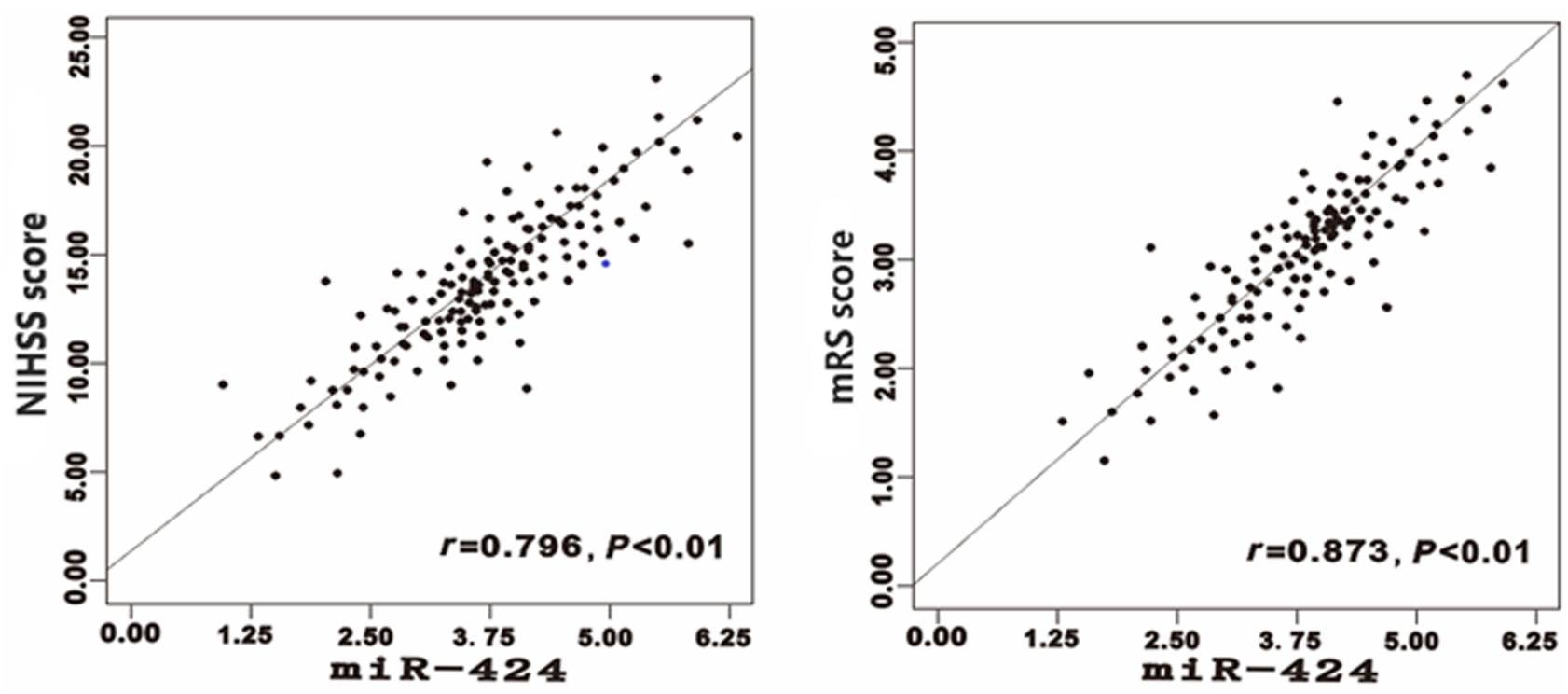

Figure 7 Correlations between the expression of serum miR-424 and NIHSS, mRS score in patients with AIS.

Abbreviations: AIS, acute ischemic stroke; NIHSS, National Institutes of Health Stroke Scale; mRS, modified Rankin scale.

diagnostic value for AIS. Li et al's research ${ }^{10}$ showed higher expression of serum miR-424 in AIS patients, and its expression was linked with the severity of the disease; suggests that miR-424 can be a biomarker for the diagnosis of AIS and can provide clues for the individualized treatment of AIS. At present, the most commonly used stroke scale globally is the NIHSS score, which can be used not only to evaluate neurological impairment of stroke patients, but also to regularly evaluate the treatment effect on stroke patients. The degree of disability or independent ability in daily activities of post-stroke patients were evaluated by mRS. In this study, the expression of serum miR-185 and miR-424 is positively related to the NIHSS score and mRS score. The increased expressions of serum miR-185 and miR-424 are closely linked with the severity of neurological impairment and poor prognosis in AIS patients. They can become potential markers for predicting poor prognosis in AIS patients as well.

\section{Conclusion}

There are two factors related to the up-regulated expression of serum miR-185 and miR-424, one is the severity degree of neurological impairment of patients with AIS and the other is their prognosis. These two combined indicators can contribute to predicting the prognosis of AIS patients. However, the specific mechanism of serum miR-185 and miR-424 involved in AIS remains unclear. We will investigate further in the future experiment. Furthermore, more tests on other classical miRNAs as the contrast for highlighting the specificity of miR-182 and miR-424 are also necessary and beneficial in future study.

\section{Data Sharing Statement}

All data generated or analyzed during this study are included in this published article.

\section{Ethical Statement}

The authors are accountable for all aspects of the work in ensuring that questions related to the accuracy or integrity of any part of the work are appropriately investigated and resolved. The study was conducted in accordance with the Declaration of Helsinki (as revised in 2013).

\section{Acknowledgments}

We sincerely thank graduate students named "Haitao Hu" and "Chunzheng Zhong" from Department of Neurology, People's Hospital of Danzhou for helping us in data collection and collation. Chunxuan Guo and Yanping Yao are co-first authors for this study.

\section{Author Contributions}

$\mathrm{HC}$ conceived study design; $\mathrm{CG}$ and $\mathrm{YY}$ conceived the content concept; QL and YG performed the data collection, extraction and analyzed the data. CG, YY and HC interpreted and reviewed the data and drafts. CG and $\mathrm{HC}$ reviewed the final draft. All authors were involved in literature search, writing the paper and had final approval of the 
submitted and published versions. All authors contributed to data analysis, drafting or revising the article, have agreed on the journal to which the article will be submitted, gave final approval of the version to be published, and agree to be accountable for all aspects of the work.

\section{Funding}

The authors declare that there are no sources of funding to be acknowledged.

\section{Disclosure}

The authors declare that they have no competing interests.

\section{References}

1. Ideta TR, Lim E, Nakagawa K, Koenig MA. Racial and ethnic disparities in hospital mortality among ischemic stroke patients in Hawaii. J Stroke Cerebrovasc Dis. 2018;27:1458-1465. doi:10.1016/ j.jstrokecerebrovasdis.2017.12.042

2. Rosengren A, Giang KW, Lappas G, Jern C, Toren K, Bjorck L. Twenty-four-year trends in the incidence of ischemic stroke in Sweden from 1987 to 2010. Stroke. 2013;44:2388-2393. doi:10.1161/STROKEAHA.113.001170

3. Zheng G, Chen B, Fang Q, et al. Primary prevention for risk factors of ischemic stroke with Baduanjin exercise intervention in the community elder population: study protocol for a randomized controlled trial. Trials. 2014;15:113. doi:10.1186/1745-6215-15-113

4. Yao XY, Lin Y, Geng JL, et al. Age- and gender-specific prevalence of risk factors in patients with first-ever ischemic stroke in China. Stroke Res Treat. 2012;2012:136398. doi:10.1155/2012/136398

5. Zhang Q, Gao T, Luo Y, et al. Transient focal cerebral ischemia/ reperfusion induces early and chronic axonal changes in rats: its importance for the risk of Alzheimer's disease. PLoS One. 2012;7: e33722. doi:10.1371/journal.pone.0033722

6. Jolana L, Kamil D. The role of microRNA in ischemic and hemorrhagic stroke. Curr Drug Deliv. 2017;14:816-831.

7. Choi GH, Ko KH, Kim JO, et al. Association of miR-34a, miR-130a, miR-150 and miR-155 polymorphisms with the risk of ischemic stroke. Int J Mol Med. 2016;38:345-356. doi:10.3892/ijmm.2016.2609

8. Jin F, Xing J. Circulating miR-126 and miR-130a levels correlate with lower disease risk, disease severity, and reduced inflammatory cytokine levels in acute ischemic stroke patients. Neurol Sci. 2018;39:1757-1765. doi:10.1007/s10072-018-3499-7

9. Xiang Y, Zhang Y, Xia Y, Zhao H, Liu A, Chen Y. LncRNA MEG3 targeting miR-424-5p via MAPK signaling pathway mediates neuronal apoptosis in ischemic stroke. Aging. 2020;12:3156-3174. doi:10.1 8632/aging. 102790
10. Li G, Ma Q, Wang R, et al. Diagnostic and immunosuppressive potential of elevated Mir-424 levels in circulating immune cells of ischemic stroke patients. Aging Dis. 2018;9:172-181. doi:10.14336/ AD.2017.0602

11. Li SH, Su SY, Liu JL. Differential regulation of microRNAs in patients with ischemic stroke. Curr Neurovasc Res. 2015;12:214-221. doi:10.2174/1567202612666150605121709

12. Zhao H, Wang J, Gao L, et al. MiRNA-424 protects against permanent focal cerebral ischemia injury in mice involving suppressing microglia activation. Stroke. 2013;44:1706-1713. doi:10.1161/ STROKEAHA.111.000504

13. Liu L, Chen W, Zhou H, et al. Chinese Stroke Association guidelines for clinical management of cerebrovascular disorders: executive summary and 2019 update of clinical management of ischaemic cerebrovascular diseases. Stroke Vasc Neurol. 2020;5:159-176. doi:10.1136/ svn-2020-000378

14. Zhang Y, Chen Z, Tang Y, et al. Association between procalcitonin levels and carotid atherosclerosis in acute ischemic stroke patients. Int $J$ Neurosci. 2018;128:237-242. doi:10.1080/00207454.20 17.1387114

15. Dewdney B, Trollope A, Moxon J, Thomas Manapurathe D, Biros E, Golledge J. Circulating MicroRNAs as biomarkers for acute ischemic stroke: a systematic review. $J$ Stroke Cerebrovasc Dis. 2018;27:522-530. doi:10.1016/j.jstrokecerebrovasdis.2017.09.058

16. Gorur A, Celik A, Yildirim DD, Gundes A, Tamer L. Investigation of possible effects of microRNAs involved in regulation of lipid metabolism in the pathogenesis of atherosclerosis. Mol Biol Rep. 2019;46:909-920. doi:10.1007/s11033-018-4547-3

17. $\mathrm{Lu} \mathrm{Y,} \mathrm{Thavarajah} \mathrm{T}, \mathrm{Gu} \mathrm{W}$, Cai J, Xu Q. Impact of miRNA in atherosclerosis. Arterioscler Thromb Vasc Biol. 2018;38:e159-e170. doi:10.1161/ATVBAHA.118.310227

18. Xu W, Gao L, Zheng J, et al. The roles of MicroRNAs in stroke: possible therapeutic targets. Cell Transplant. 2018;27:1778-1788. doi:10.1177/0963689718773361

19. Laffont B, Rayner KJ. MicroRNAs in the pathobiology and therapy of atherosclerosis. Can J Cardiol. 2017;33:313-324. doi:10.1016/j. cjca.2017.01.001

20. Gacon J, Badacz R, Stepien E, et al. Diagnostic and prognostic micro-RNAs in ischaemic stroke due to carotid artery stenosis and in acute coronary syndrome: a four-year prospective study. Kardiol Pol. 2018;76:362-369. doi:10.5603/KP.a2017.0243

21. Wu J, Fan CL, Ma LJ, et al. Distinctive expression signatures of serum microRNAs in ischaemic stroke and transient ischaemic attack patients. Thromb Haemost. 2017;117:992-1001. doi:10.1160/TH1608-0606

22. Jin F, Xing J. Circulating pro-angiogenic and anti-angiogenic microRNA expressions in patients with acute ischemic stroke and their association with disease severity. Neurol Sci. 2017;38:2015-2023. doi:10.1007/s10072-017-3071-x
International Journal of General Medicine

\section{Publish your work in this journal}

The International Journal of General Medicine is an international, peer-reviewed open-access journal that focuses on general and internal medicine, pathogenesis, epidemiology, diagnosis, monitoring and treatment protocols. The journal is characterized by the rapid reporting of reviews, original research and clinical studies across all disease areas. The manuscript management system is completely online and includes a very quick and fair peer-review system, which is all easy to use. Visit http://www.dovepress.com/ testimonials.php to read real quotes from published authors. 